\title{
Coexpression of ATP-binding cassette proteins ABCG5 and ABCG8 permits their transport to the apical surface
}

\author{
Gregory A. Graf, ${ }^{1}$ Wei-Ping Li, ${ }^{2}$ Robert D. Gerard,${ }^{1}$ Ingrid Gelissen, ${ }^{1}$ Ann White,${ }^{1}$ \\ Jonathan C. Cohen, ${ }^{1,3}$ and Helen H. Hobbs ${ }^{1}$
}

${ }^{1}$ McDermott Center for Human Growth and Development, Department of Molecular Genetics,
${ }^{2}$ Department of Cell Biology, and
${ }^{3}$ Center for Human Nutrition, University of Texas Southwestern Medical Center at Dallas, Dallas, Texas, USA

\begin{abstract}
Mutations in either ATP-binding cassette (ABC) G5 or ABCG8 cause sitosterolemia, an autosomal recessive disorder of sterol trafficking. To determine the site of action of ABCG5 and ABCG8, we expressed recombinant, epitope-tagged mouse ABCG5 and ABCG8 in cultured cells. Both ABCG5 and ABCG8 underwent $\mathrm{N}$-linked glycosylation. When either protein was expressed individually in cells, the N-linked sugars remained sensitive to Endoglycosidase H (Endo H). When ABCG5 and ABCG8 were coexpressed, the attached sugars were Endo H-resistant and neuraminidase-sensitive, indicating that the proteins were transported to the trans-Golgi complex. The mature, glycosylated forms of ABCG5 and ABCG8 coimmunoprecipitated, consistent with heterodimerization of these two proteins. The Endo H-sensitive forms of ABCG5 and ABCG8 were confined to the endoplasmic reticulum (ER), whereas the mature forms were present in non-ER fractions in cultured hepatocytes. Immunoelectron microscopy revealed ABCG5 and ABCG8 on the plasma membrane of these cells. In polarized WIF-B cells, recombinant ABCG5 localized to the apical (canalicular) membrane when coexpressed with ABCG8, but not when expressed alone. To our knowledge this is the first direct demonstration that trafficking of an $\mathrm{ABC}$ half-transporter to the cell surface requires the presence of its dimerization partner.
\end{abstract}

J. Clin. Invest. 110:659-669 (2002). doi:10.1172/JCI200216000.

\section{Introduction}

Mutations in the ATP-binding cassette (ABC) halftransporters $A B C G 5$ and $A B C G 8$ cause sitosterolemia $(1,2)$, a rare autosomal recessive disorder characterized by elevated plasma levels of both plant and animal sterols $(3,4)$. Normal individuals absorb less than 5\% of dietary sitosterol, the major plant sterol, and efficiently excrete plant sterols into bile $(5,6)$. Plasma sitosterol concentrations rarely exceed $1 \mathrm{mg} / \mathrm{dl}$, even in individuals with very high intakes of dietary sitosterol. In contrast, sitosterolemic subjects absorb about 15-20\% of dietary sitosterol and have a profoundly reduced capacity to excrete sitosterol into bile. Consequently, sitosterol accumulates in the blood and body tissues of patients with this genetic disease $(5,7-11)$. A greater fraction of both animal- and plant-derived sterols are

Received for publication May 24, 2002, and accepted in revised form July 9, 2002.

Address correspondence to: Helen H. Hobbs, Department of Molecular Genetics, University of Texas Southwestern Medical Center at Dallas, 5323 Harry Hines Boulevard, Dallas, Texas 75390-9046, USA. Phone: (214) 648-6724; Fax: (214) 648-7539; E-mail: Helen.Hobbs@UTSouthwestern.edu.

Conflict of interest: No conflict of interest has been declared. Nonstandard abbreviations used: ATP-binding cassette (ABC); endoplasmic reticulum (ER); aminopeptidase N (APN); hemagglutinin (HA); polyclonal antibody (pAb); peptide $\mathrm{N}$-glycosidase F (PNGase F); Endoglycosidase H (Endo H); LDL receptor-related protein (LRP); $\beta$-galactosidase ( $\beta$-gal). absorbed from the diet in affected individuals. The increased fractional absorption and reduced biliary excretion of cholesterol in sitosterolemia results in hypercholesterolemia and premature coronary artery disease $(4,11)$. The phenotype of sitosterolemia suggests that ABCG5 and ABCG8 limit intestinal absorption and promote biliary excretion of neutral sterols.

$\mathrm{ABC}$ transporters share a common molecular architecture that includes two nucleotide-binding folds and two transmembrane domains containing 6-11 membrane-spanning $\alpha$-helices $(12,13)$. ABC transporters are organized as full transporters containing two transmembrane domains and two nucleotide-binding folds, or as half-transporters that form homo- or heterodimers (14). ABCG5 and ABCG8 are members of the $\mathrm{G}$ subfamily of $\mathrm{ABC}$ transporters, which are predicted to contain a single magnesium-dependent ATP catalytic domain $\mathrm{N}$-terminal to six transmembrane segments (1). Mutations in either ABCG5 or ABCG8 cause an identical phenotype, which is consistent with these two gene products functioning as a heterodimer $(1,15)$, though this has yet to be demonstrated.

Most ABC half-transporters reside in intracellular membranes (14). The best-characterized mammalian half transporters are TAP1 and TAP2 (ABCB2 and $A B C B 3)$, which are endoplasmic reticulum (ER) resident proteins that transport peptides from the cytosol into the lumen of this compartment (16). All four ABC 
half-transporters in the D subfamily are associated with the peroxisomal membrane (17). The Drosophila ABCG transporters white, brown and scarlet are located within membranes of intracellular pigment granules (18). The only mammalian $\mathrm{ABC}$ half-transporter that has been localized to the plasma membrane is the multidrug resistance protein ABCG2 (BCRP), which is associated with the canalicular membrane in human liver (19). ABCG5 and $A B C G 8$ are most highly expressed in the enterocytes of the intestine and hepatocytes of the liver (20), but the subcellular location of the two proteins is not known.

In this paper, we have examined the cellular itinerary and fate of recombinant, epitope-tagged mouse ABCG5 and ABCG8 in three cell types: CHO-K1 cells, cultured rat hepatocytes (CRL-1601 cells), and polarized WIF-B cells $(21,22)$. We provide evidence that ABCG5 and ABCG8 are physically associated in cells and that they chaperone each other out of the ER to the plasma membrane. In polarized WIF-B cells, the transporters colocalized with a resident apical membrane protein, aminopeptidase $\mathrm{N}$ (APN), on the canalicular membrane. Our data are consistent with ABCG5 and ABCG8 forming a heterodimer in the ER prior to being transported to apical membranes.

\section{Methods}

Development of epitope-tagged recombinant $A B C G 5$ and $A B C G 8$. A 105-bp fragment encoding three copies of a myc epitope (EQKLISEEDLN) (23) and a 93-bp fragment containing three copies of a hemagglutinin (HA) epitope (YPYDVPDYAG) (24) were added to the $3^{\prime}$ ends of the murine ABCG5 and ABCG8 CDNAs by overlap PCR. The ABCG5-myc, ABCG5-HA, ABCG8-myc, and ABCG8-HA cDNAs were individually cloned into pcDNA3.1(+) and pcDNA3.1/Zeo (Invitrogen Corp., Carlsbad, California, USA). The fidelity of each construct was confirmed by DNA sequencing. The initial ABCG8-myc construct used in these studies had a proline at amino acid residue 220. Subsequently, Yu et al. (25) reported a sequence containing arginine at this position. Therefore an expression construct that encoded an arginine at residue 220 was also made.

Cell culture. CHO-K1 cells (American Type Culture Collection, Manassas, Virginia, USA) were cultured in Ham's F12/DMEM 50\% (vol/vol) medium containing $5 \%$ (vol/vol) FBS, $100 \mathrm{U} / \mathrm{ml}$ penicillin, and $100 \mu \mathrm{g} / \mathrm{ml}$ streptomycin (Life Technologies Inc., Grand Island, New York, USA) in a humidified incubator $\left(8.8 \% \mathrm{CO}_{2}\right)$. CRL-1601 cells (American Type Culture Collection) were cultured in DMEM (glucose, $1 \mathrm{~g} / \mathrm{l}$ ) containing $10 \%$ (vol/vol) FBS, $100 \mathrm{U} / \mathrm{ml}$ penicillin, and $100 \mu \mathrm{g} / \mathrm{ml}$ streptomycin in a humidified incubator $\left(5 \% \mathrm{CO}_{2}\right)$. WIF-B cells were a generous gift from Ann Hubbard (Johns Hopkins University, Baltimore, Maryland, USA) and were cultured as previously described (22).

Immunoprecipitation of epitope-tagged ABCG5 and ABCG8. Epitope-tagged ABCG5 and ABCG8 expression constructs were transiently transfected either alone or together into CHO-K1 cells using FuGene (Roche Molecular Biochemicals, Mannheim, Germany) according to the manufacturer's protocol. Immunoprecipitations were performed as previously described (26) with the following modification: Incubations of primary antibodies with cell lysates were limited to 6 hours. Mouse anti-HA antibody (12CA5) and anti-myc antibody (9E10; Roche Molecular Biochemicals) were used to immunoprecipitate ABCG8 and ABCG5, respectively.

SDS-PAGE and immunoblot analysis of ABCG5 and $A B C G 8$. The protein concentrations of cell lysates and fractions were determined by Bio-Rad Dc assay according to the manufacturer's protocol (Bio-Rad Laboratories Inc., Hercules, California, USA). Proteins were size-fractionated on either $8 \%$ or $4-20 \%$ gradient (wt/vol) SDS-polyacrylamide gels and transferred to nitrocellulose membranes. Membranes were immunoblotted as described (27) using polyclonal antibodies (pAb's) directed against the myc (A14) and HA (Y11) epitopes (Santa Cruz Biotechnology Inc., Santa Cruz, California, USA).

Pulse-chase analysis of $A B C G 5$ and $A B C G 8$ synthesis in cultured cells. CHO-K1 cells were transfected with ABCG5myc and ABCG8-HA expression plasmids either alone or together. After 48 hours, the cells were washed twice with PBS $\left(37^{\circ} \mathrm{C}\right)$ and incubated in methionine- and cysteinefree DMEM for 2 hours. Newly translated proteins were metabolically labeled for 15 minutes with methionineand cysteine-free DMEM containing $100 \mu \mathrm{Ci} / \mathrm{ml}\left[{ }^{35} \mathrm{~S}\right]-$ Met/Cys (Perkin-Elmer Life Sciences Inc., Boston, Massachusetts, USA). Cells were washed twice with PBS $\left(37^{\circ} \mathrm{C}\right)$ and incubated with DMEM for up to 48 hours. Cell lysates were prepared and immunoprecipitations were conducted as described above; primary antibodies were incubated with lysates for 18 hours at $22^{\circ} \mathrm{C}$. The immunoprecipitates were subjected to SDS-PAGE and the gels fixed for 30 minutes ( $40 \%$ methanol, $10 \%$ acetic acid). Signals from the labeled proteins were amplified by fluorography (30 minutes, $1 \mathrm{M}$ salicylate), and the gels were dried and exposed to autoradiography film $\left(-80^{\circ} \mathrm{C}, 8-48\right.$ hours). The relative signal intensities were determined by densitometry (Eagle Eye II; Stratagene, La Jolla, California, USA). The values obtained for each time point were corrected for background signal, log-transformed, and used to calculate the $t_{1 / 2}$ of each protein by linear regression using the least-squares method.

Analysis of glycosylation of $A B C G 5$ and $A B C G 8$. The glycosylation state of ABCG5-myc and ABCG8-HA was analyzed in transiently transfected CHO-K1 cells. Cells were cultured in 10-cm dishes and transfected, and the proteins were immunoprecipitated as described above. Immunoprecipitated proteins were recovered from protein A-agarose beads by heating in 0.1\% SDS, $1 \mathrm{mM}$ $\beta$-mercaptoethanol $\left(95^{\circ} \mathrm{C}, 5\right.$ minutes). Protein A-agarose was removed, and the supernatants were transferred to new tubes. The immunoprecipitated proteins were incubated in the presence or absence of $10 \mathrm{U}$ of peptide N-glycosidase F (PNGase F); Calbiochem-Novabiochem 
Corp., San Diego, California, USA), 5 U of Endoglycosidase $\mathrm{H}$ (Endo H; Roche Molecular Biochemicals), $50 \mathrm{mU}$ neuraminidase (Roche Molecular Biochemicals), or 2.5 $\mathrm{mU}$ O-glycosidase (Roche Molecular Biochemicals) for 2 hours at $37^{\circ} \mathrm{C}$ following digestion with neuraminidase according to the manufacturers' protocols. Samples were subjected to SDS-PAGE and immunoblot analysis.

Generation of cell lines stably expressing $A B C G 5$-myc and ABCG5-myc plus ABCG8-HA. CRL-1601 cells were transfected at $60 \%$ confluence as described for CHO-K1 cells. Cells were selected in medium containing $700 \mu \mathrm{g} / \mathrm{ml}$ G418 sulfate and $700 \mu \mathrm{g} / \mathrm{ml}$ Zeocin (Invitrogen Corp.). Cells were grown under continuous selection, and single colonies were selected, subcultured, and screened for expression of ABCG5-myc and ABCG8-HA by immunoblot analysis as described above.

Cell fractionation. Cell lysates were fractionated by density gradient centrifugation in Nycodenz (Sigma, St. Louis, Missouri, USA) to separate the ER from other organelles (28). Fractions $(n=36)$ were collected after tube puncture. Protein sample buffer was added to a final concentration of $1 \times$, and $100 \mu$ l of each odd-numbered fraction was subjected to SDS-PAGE and immunoblot analysis. The separation of the ER from other intracellular compartments was confirmed by immunoblot analysis of proteins associated with the ER (calnexin; StressGen Biotechnologies Corp., Victoria, British Columbia, Canada) with the Golgi complex (mannosidase II; Kelly Mormen, University of Georgia, Athens, Georgia, USA), with the endosomes (Rab11; Santa Cruz Biotechnology Inc.), and with the plasma membrane and endosomes (LDL receptor-related protein [LRP] $85-\mathrm{kDa}$ form) (29).

Immunofluorescence and immunoelectron microscopy. The subcellular localization of ABCG5-myc and ABCG8-HA in cultured cells was determined by immunocytochemical analysis as described previously (30). Cells were imaged by confocal microscopy using a Leica TCS SP microscope (Leica Microsystems Inc., Heidelberg, Germany).

For immunoelectron microscopy, cells were cultured in $100-\mathrm{mm}$ dishes to $95 \%$ confluence and fixed with 3\% (wt/vol) paraformaldehyde and $0.2 \%$ glutaraldehyde in PBS (100 mM sodium phosphate [pH 7.4] $0.15 \mathrm{M}$ $\mathrm{NaCl}, 4 \mathrm{mM} \mathrm{KCl}, 2 \mathrm{mM} \mathrm{MgCl}_{2}$ ) for 5 minutes at $37^{\circ} \mathrm{C}$, followed by fixation in the same fixative for 25 minutes $\left(22^{\circ} \mathrm{C}\right)$. After brief rinses with PBS, the samples were immersed with PBS containing $50 \mathrm{mM} \mathrm{NH}_{4} \mathrm{Cl}$ for 30 minutes and enclosed in 2\% agarose. The cell pellets were immersed in $2 \mathrm{M}$ sucrose containing $15 \%$ polyvinylpyrrolidone $(10 \mathrm{kDa})$ and processed for frozen ultrathin sectioning according to the method of Tokuyasu (31). Cryosections were conditioned on droplets of conditioning buffer (PBS with 1\% BSA, $0.01 \%$ Triton X-100, and $0.01 \%$ Tween-20) for 20 minutes and then incubated for 2 hours in the presence of either anti-myc pAb or anti-HA pAb $(10 \mu \mathrm{g} / \mathrm{ml}$ in conditioning buffer). The IgG was localized by incubating sections for 1 hour with $10 \mathrm{~nm}$ gold-labeled goat anti- rabbit IgG diluted 1:40 in conditioning buffer. Electron micrographs were taken with a JEOL 1200 electron microscope (JEOL USA Inc., Peabody, Massachusetts, USA) operating at $80 \mathrm{kV}$.

Generation of recombinant adenoviruses expressing ABCG5myc and $A B C G 8$. Recombinant adenoviral vectors containing ABCG5, ABCG5-myc, ABCG8, and ABCG8-HA were generated by in vitro cre-lox recombination (32). The adenovirus particles were used to express ABCG5myc and untagged ABCG8 in WIF-B cells that were cultured as described above. Ten days following plating, cells were infected with $1 \times 10^{9}$ particles of virus expressing $\beta$-galactosidase ( $\beta$-gal) ABCG5-myc, ABCG8, or both ABCG-myc and ABCG8 cDNAs.

\section{Results}

Coexpression of ABCG5 and ABCG8 induces posttranslational modification of the proteins. To examine the size, posttranslational processing, and half-lives of ABCG5 and ABCG8, the cDNAs encoding the two proteins were modified to contain epitope tags at the C-termini and then expressed independently in CHO-K1 cells (Figure 1, left). Transfected cells were labeled with $\left[{ }^{35} \mathrm{~S}\right]-\mathrm{Met} / \mathrm{Cys}$ for 15 minutes and then chased with excess Met/Cys for the indicated time periods. ABCG5myc and ABCG8-HA were immunoprecipitated from cell lysates using mAb's to the epitope tags. At the 0 time point, three proteins of $65 \mathrm{kDa}, 70 \mathrm{kDa}$, and 73 $\mathrm{kDa}$ were observed for ABCG5, and two proteins of 66 and $68 \mathrm{kDa}$ for ABCG8-HA. The $t_{1 / 2}$ of both ABCG5myc and ABCG8-HA was approximately 3 hours. When ABCG5-myc and ABCG8-HA were coexpressed (Figure 1, right), a series of higher-molecular weight proteins $(90-100 \mathrm{kDa}$ for ABCG5-myc and about 78 $\mathrm{kDa}$ for ABCG8-HA) appeared at 2 hours and remained detectable at 24 hours, when the lowermolecular weight proteins were barely visible. No higher-molecular weight proteins were detected with longer exposures in cells expressing either ABCG5 or ABCG8 alone (data not shown). These results suggest that both ABCG5 and ABCG8 undergo an initial processing event that is not dependent on the presence of the other protein. After 1 to 2 hours, the proteins undergo further modifications, which are contingent on the expression of both proteins. The sequential appearance of higher-molecular weight proteins is consistent with a precursor-product relationship.

The identity of a $48-\mathrm{kDa}$ protein that immunoprecipitated with the anti-HA antibody and decreased in intensity over the course of the experiment is not known. A protein of this size was detected in CHO-K1 cells expressing ABCG8-HA, but not when this protein was expressed in CRL-1601 cells.

Coexpression of $A B C G 5$ and $A B C G 8$ is required for trafficking out of the ER. To determine whether the higher-molecular weight forms of ABCG5-myc and ABCG8-HA observed in Figure 1 were due to glycosylation, we immunoprecipitated the proteins and then subjected them to treatment with different 


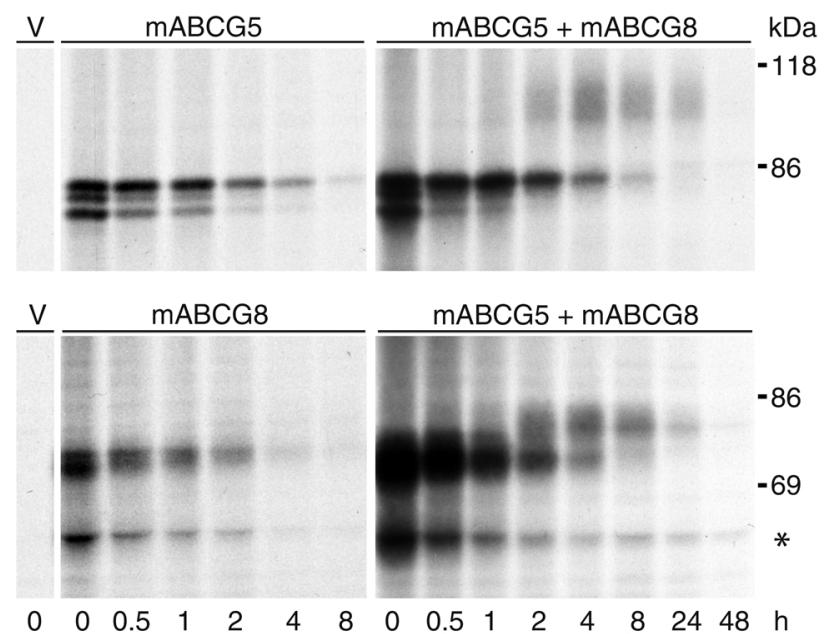

Figure 1

Pulse-chase analysis of epitope-tagged ABCG5 and ABCG8 in transiently transfected $\mathrm{CHO}-\mathrm{K} 1$ cells. Cells were transfected with empty plasmids (V), ABCG5-myc, ABCG8-HA, or ABCG5-myc and ABCG8$\mathrm{HA}$. Forty-eight hours following transfection, cells were washed with PBS, incubated in methionine- and cysteine-free medium for 2 hours, and then incubated in methionine- and cysteine-free medium supplemented with $\left.100 \mu \mathrm{Ci} / \mathrm{ml}{ }^{35} \mathrm{~S}\right]-M e t / C y s$ for 15 minutes. Cells were washed with PBS and incubated in serum-free medium for the indicated times. Cell lysates were prepared, and anti-myc mAb was used to immunoprecipitate $A B C G 5$-myc in cells expressing ABCG5-myc alone or ABCG5-myc in the presence of ABCG8-HA (top panels). Anti-HA mAb was used to immunoprecipitate $A B C G 8$ in cells expressing $\mathrm{ABCG} 8-\mathrm{HA}$ alone or $\mathrm{ABCG} 8-\mathrm{HA}$ in the presence of $\mathrm{ABCG}$-myc (bottom panels). Immunoprecipitated proteins were resolved by SDSPAGE (4-20\%) and visualized by fluorography (exposure: 8 hours, left; 36 hours, right). This experiment was repeated four times with similar results. *unidentified $48 \mathrm{kDa}$ band observed in $\mathrm{CHO}-\mathrm{K} 1$ cells transfected with ABCG8.

endoglycosidases (Figure 2). In cells expressing ABCG5-myc or ABCG8-HA alone, the higher-molecular weight forms of the proteins were sensitive to PNGase $\mathrm{F}$, which removes all N-linked sugars, and to Endo $\mathrm{H}$, which removes high-mannose sugars that have not yet undergone full maturation. Mouse ABCG8 has one canonical site for the addition of $\mathrm{N}$-linked sugars at residue 619, and ABCG5 has three potential sites for addition of $\mathrm{N}$-linked sugars (residues 440,585, and 592), but one of these sites is predicted to be within a few residues of the first membrane-spanning domain and is not conserved in the human protein (Figure 2, bottom). The stepwise increases in molecular masses of these proteins in transfected $\mathrm{CHO}-\mathrm{K} 1$ cells are consistent with the covalent addition of one N-linked sugar to ABCG8-HA and two N-linked sugars to ABCG5-myc (Figure 1). Treatment with neuraminidase or with neuraminidase plus O-glycosidase did not change the migration of either ABCG5-myc or ABCG8-HA when the proteins were expressed individually. The LDL receptor was examined in the same experiment, and the expected approximately $40-\mathrm{kDa}$ decrease in apparent molecular mass following treatment with O-glycosidase was detected (data not shown) (33). Thus, when ABCG5-myc or ABCG8-HA was expressed alone, $\mathrm{N}$-linked sugars were added in the ER but the proteins did not appear to traffic to the Golgi complex.

Coexpression of ABCG5-myc and ABCG8-HA resulted in the appearance of additional higher-molecular weight forms, as described above (Figure 1). Immunoblotting revealed that in the steady state the higher-molecular weight forms of the proteins predominated, especially for ABCG8-HA. The more slowly migrating proteins collapsed into a single protein upon treatment with PNGase F but were resistant to treatment with Endo H, indicating that the more slowly migrating forms of the proteins had reached the medial Golgi complex, whereas the lower-molecular weight forms were retained in the ER. Neuraminidase, which removes sialic acid residues added in the transGolgi complex (34), caused a slight but consistent reduction in the apparent molecular mass of the larger forms of ABCG5-myc and ABCG8-HA. No further changes in protein mass were apparent with O-glycosidase treatment. Taken together, these results indicated that ABCG5-myc and ABCG8-HA were retained in the ER unless expressed together, and they suggested that ABCG5 and ABCG8 form a heterodimer in order to exit the ER.

Recombinant ABCG5 and ABCG8 coimmunoprecipitate in CHO-K1 cells. To determine whether ABCG5 and ABCG8 are physically associated in cells, we expressed myctagged ABCG5 and HA-tagged ABCG8 alone or together in CHO-K1 cells (Figure 3a). The anti-myc antibody precipitated a protein of the size expected for ABCG5myc in cells expressing both ABCG5-myc and ABCG8$\mathrm{HA}$, but not in cells expressing ABCG8-HA alone; similarly, the anti-HA antibody immunoprecipitated a protein of the size expected for ABCG8-HA in cells expressing both transporters but not in cells expressing only ABCG5-myc. More than $95 \%$ of each protein was immunoprecipitated by mAb's to their respective epitope tags. ABCG8-HA coimmunoprecipitated with ABCG5-myc and ABCG5-myc coimmunoprecipitated with ABCG8-HA in cells expressing both proteins.

To determine whether the association between ABCG5-myc and ABCG8-HA occurred before or after cell lysis, cells were first transfected independently with ABCG5-myc, ABCG5-HA, ABCG8-myc, or ABCG8-HA (Figure $3 \mathrm{~b}$ ). Lysates from the independently transfected cells were mixed (5\&5, 5-myc and 5-HA; 5\&8, 5-myc and 8-HA; 8\&8, 8-myc and 8-HA) and then subjected to immunoprecipitation and immunoblot analysis. The anti-myc mAb failed to immunoprecipitate the HAtagged transporters, and the myc-tagged transporters did not coimmunoprecipitate with the anti-HA mAb. Thus, the association of ABCG5-myc and ABCG8-HA did not occur after cell lysis.

To test for the formation of homo-oligomeric complexes, we cotransfected cells with ABCG5-myc and ABCG5-HA (5+5), with ABCG5-myc and ABCG8-HA 
$(5+8)$, or with ABCG8-myc and ABCG8-HA $(8+8$, Figure $3 \mathrm{~b})$. A small fraction of ABCG5 and a significant fraction of ABCG8 coimmunoprecipitated in cells expressing both the myc- and the HA-tagged forms of the same proteins. In both cases, only the lower-molecular weight forms of the proteins were detected, indicating that if homodimers form, they are retained in the ER. In contrast, both the lower- and the higher-molecular weight forms were observed in cells expressing both ABCG5myc and ABCG8-HA and coimmunoprecipitated with antibodies to either epitope. These results are consistent with the half-transporters forming heterodimers and then trafficking to the Golgi complex.

To ensure that the substitution of proline for arginine at codon 220 of ABCG8 did not affect the association or trafficking of ABCG8, the pulse-chase, endoglycosidase digestion, and coimmunoprecipitation experiments were repeated using an expression construct encoding an arginine at residue 220 , and identical results were obtained (data not shown).

ABCG8 expression affects the trafficking of ABCG5. To trace the intracellular trafficking of ABCG5 and ABCG8 in a physiologically relevant cell type, rat hepatocyte-derived cell lines (CRL-1601) expressing either recombinant ABCG5-myc alone or ABCG5-myc and ABCG8-HA together were selected and subcloned. No endogenous ABCG5 or ABCG8 mRNA was detected by Northern blotting in these cells (data not shown). Steady-state levels of ABCG5-myc and ABCG8-HA were analyzed by immunoblotting in both cell lines (Figure 4a). A single protein of $68 \mathrm{kDa}$ was detected in cells expressing ABCG5-myc alone, whereas additional higher-molecular weight forms of the protein $(89-100 \mathrm{kDa})$ were present in cells expressing both ABCG5-myc and ABCG8-HA.
To determine the subcellular location of the half-transporters in the steady state, lysates from cells expressing ABCG5-myc alone or ABCG5-myc plus ABCG8-HA were fractionated on Nycodenz gradients to separate the ER from other cellular compartments (Figure 4b). Immunoblot analysis of the gradient fractions indicated that the lower-molecular weight, Endo $\mathrm{H}$-sensitive forms of ABCG5-myc and ABCG8-HA comigrated with the ER resident protein calnexin. Only the higher-molecular weight, Endo $\mathrm{H}$-resistant forms were present in non-ER fractions. The higher-molecular weight forms of both ABCG5-myc and ABCG8-HA were also present in the ER fractions. The ER fractions of the gradients did not contain proteins present in the plasma membrane (LRP, 85 $\mathrm{kDa}$ ), endosomes (LRP, Rab11), or Golgi complex (p58K). $A B C G 5$ and $A B C G 8$ localize to the cell membrane of CRL1601 cells. To determine the subcellular localization of ABCG5 and ABCG8, CRL-1601 cells expressing ABCG5-myc and ABCG8-HA were grown on glass coverslips and processed for immunofluorescence and immunoelectron microscopy. Immunoreactive ABCG5 colocalized with ER resident proteins (GRP-78, calnexin) in cells expressing ABCG5 alone, but with the cell surface protein APN in cells expressing both ABCG5 and ABCG8 (data not shown). To determine whether ABCG5 and ABCG8 were on the cell surface or in closely apposed subplasmalemmal vesicles, we performed immunoelectron microscopy on cells expressing both half-transporters. Gold particles were associated with the plasma membrane and ER for ABCG5-myc (Figure 5b) and for ABCG8-HA (Figure 5c). No signal was detected in cells containing the expression vector alone (Figure 5a).

$A B C G 5$ and $A B C G 8$ traffic to the apical surface in polarized WIF-B cells. Since CRL-1601 cells are not polarized,

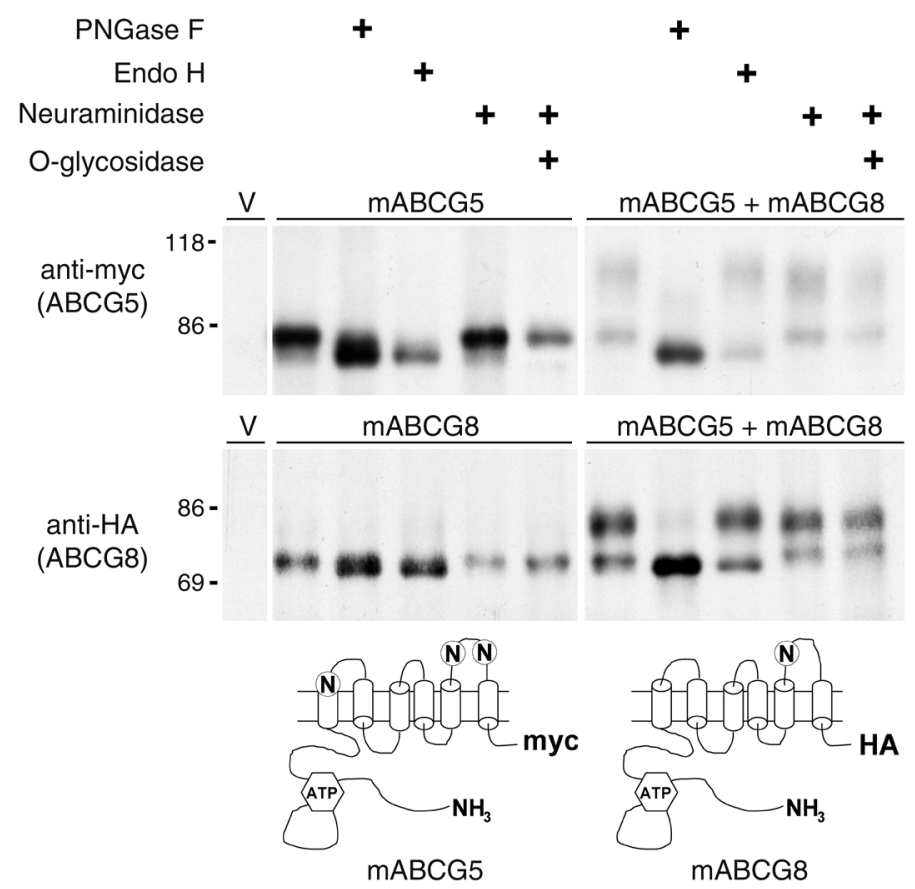

\section{Figure 2}

Endoglycosidase treatment of recombinant, epitope-tagged ABCG5 and ABCG8 in CHO-K1 cells. Cells were transfected with empty plasmid (V), ABCG5-myc, ABCG8-HA, or $A B C G 5$-myc plus ABCG8-HA. Cell lysates were prepared after 48 hours and aliquoted into two tubes. ABCG5-myc and ABCG8-HA were immunoprecipitated using mAb's directed against the myc (top) and $\mathrm{HA}$ epitopes (bottom), respectively. Protein A-agarose beads were removed, and the immunoprecipitated proteins were incubated in the presence of PNGase $F$ Endo $H$, neuraminidase, and neuraminidase followed by O-glycosidase. Proteins were precipitated with $0.015 \%$ deoxycholate and $1 \%$ trichloroacetic acid solubilized in protein sample buffer, and subjected to SDS-PAGE (8\%). Proteins were transferred to nitrocellulose membranes and subjected to immunoblot analysis using rabbit IgGs directed against the myc (ABCG5) and HA (ABCG8) epitopes. 
a

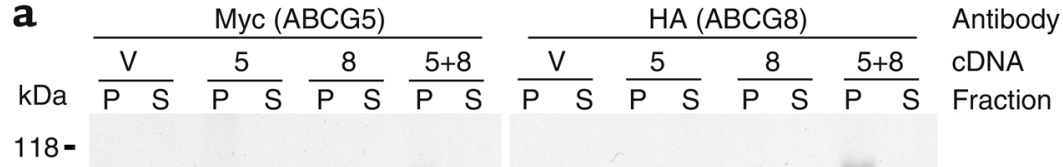

$118-$
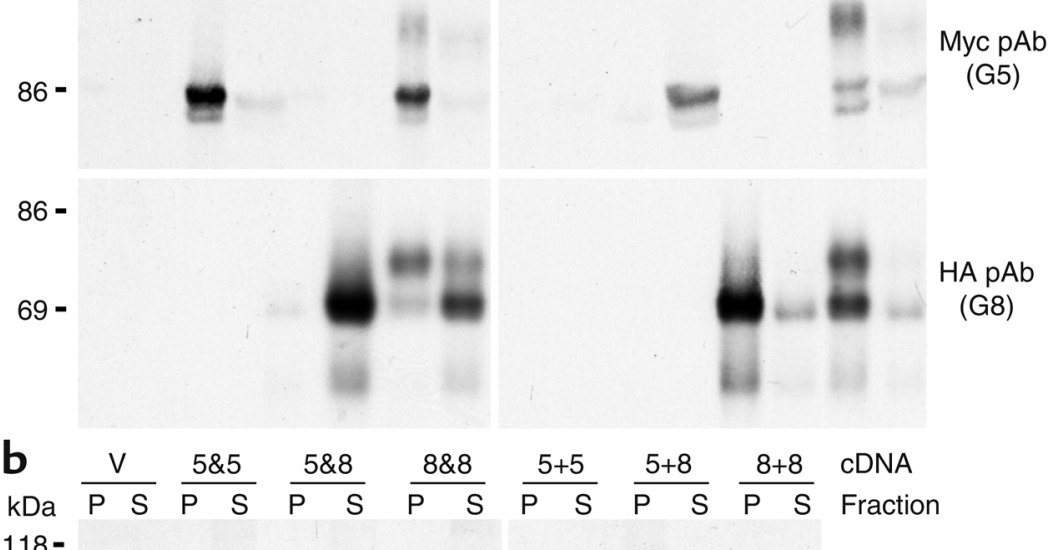

$118-$

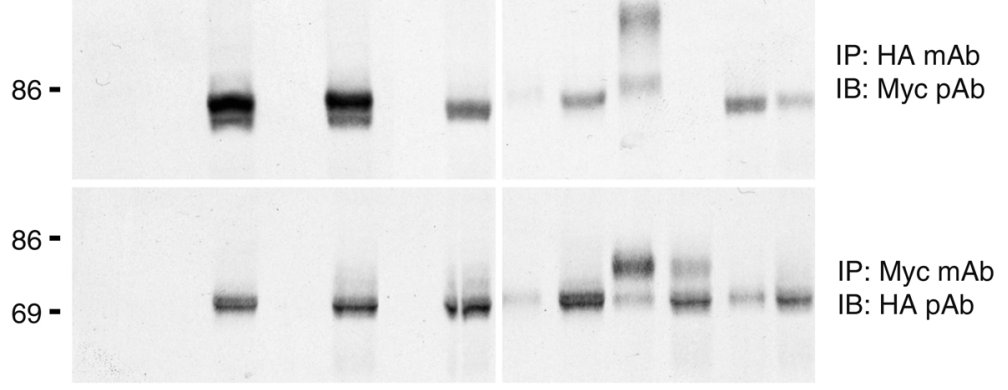

Figure 3

Coprecipitation of ABCG5 and ABCG8 in transiently transfected $\mathrm{CHO}-\mathrm{K} 1$ cells. (a) Cells were transfected with empty plasmid (V), ABCG5-myc (5), ABCG8-HA (8), or ABCG5myc plus $A B C G 8-H A(5+8)$. Forty-eight hours following transfection, cell lysates were prepared and aliquoted into two tubes. ABCG5-myc and ABCG8-HA were immunoprecipitated with mAb's directed against the myc (left) and HA epitopes (right), respectively. Duplicate aliquots $(10 \%)$ of each immunoprecipitate $(P)$ and supernatant $(S)$ were subjected to SDS-PAGE (8\%) and transferred to nitrocellulose membranes. Each membrane was subjected to immunoblot analysis using pAb's directed against the myc (G5, top) or HA (G8, bottom) epitopes. (b) Cells were transfected with ABCG5-myc, ABCG5-HA, ABCG8-myc, or ABCG8-HA (left). Cell lysates from a 60-mm dish of each of the following transfections were pooled (left): empty plasmids ( $V)$, ABCG5-myc and ABCG5-HA (5\&5), ABCG5-myc and ABCG8-HA (5\&8), and ABCG8-myc and ABCG8$\mathrm{HA}(8 \& 8)$. Two dishes were cotransfected with each of the following construct pairs (right): ABCG5-myc and ABCG5-HA (5+5), ABCG5-myc and ABCG8-HA (5+8), and $A B C G 8-m y c$ and $A B C G 8-H A(8+8)$. Lysates were aliquoted into two tubes and immunoprecipitated with mAb's to the myc (bottom) or HA epitopes (top). Duplicate aliquots of each immunoprecipitate and supernatant (10\%) were subjected to SDS-PAGE and transferred to nitrocellulose membranes. Each membrane was subjected to immunoblot analysis using PAb's against the myc (top) or HA (bottom) epitopes. IP, immunoprecipitating; IB immunoblotting.

these cells cannot be used to determine whether ABCG5 and ABCG8 are specifically targeted to the bile canalicular (apical) surface. Therefore, we obtained a polarized cell line (WIF-B) derived from a fusion between a rat hepatoma and human fibroblast cells (22). These cells form canaliculi and sort proteins in a manner similar to that observed in vivo (35) and have been used to define the subcellular localization of biliary $\mathrm{ABC}$ transporters (30). Northern blot analysis using probes specific for rat ABCG5 and ABCG8 indi-
Myc pAb

(G5)

cated that both endogenous genes were expressed in these cells (data not shown). Human chromosome 2 was not retained in this cell line (21), so these cells cannot synthesize human ABCG5 or ABCG8. To determine whether ABCG5-myc traffics beyond the ER when expressed in WIF-B cells, a pulse-chase experiment was performed in cells infected with adenoviruses encoding ABCG5-myc alone, or ABCG5-myc and ABCG8. Mature, fully glycosylated forms of ABCG5-myc were only observed when ABCG8 was coexpressed (data not shown), indicating that if heterodimers between rat and mouse ABCG5 and ABCG8 form, they are incapable of escaping the ER.

To determine the subcellular localization of ABCG5-myc in the absence or presence of ABCG8, cells were infected with adenoviruses encoding cDNAs for $\beta$-gal, ABCG5myc, or ABCG5-myc and ABCG8 and processed for immunofluorescence microscopy (Figure 6). The anti-myc antibody detected ABCG5-myc in cells expressing ABCG5-myc alone or ABCG5-myc plus ABCG8, but not in cells expressing $\beta$-gal (Figure 6 , a-c). In the absence of ABCG8, ABCG5myc colocalized with BiP, but not with basolateral (HA321) (36) or apical (APN) plasma membrane markers (Figure 6, d-1), which indicated that in WIF-B cells, as in CRL-1601 cells, ABCG5 was confined to the ER when expressed alone. In the presence of ABCG8, ABCG5-myc colocalized with the apically sorted protein APN (Figure 6, s-u). However, no colocalization was seen with HA321 or mannosidase II. A faint signal was seen on the basolateral surface of some cells (Figure 6p). Whether this represents proteins that first go to the basolateral membranes before trafficking to the apical surface or is an artifact of overexpression is not known. These results indicated that ABCG5 is sorted to the apical surface when coexpressed with ABCG8, and they suggested that ABCG5/ABCG8 heterodimers traffic to the canalicular surface of hepatocytes. While immunofluorescence microscopy does not provide sufficient resolution to exclude a subplasmalemmal localization, the results of the electron microscopy studies in the CRL-1601 cells suggest that the two proteins are located in the plasma membrane. 


\section{Discussion}

In this study we have examined the cellular itinerary and fate of recombinant, epitope-tagged ABCG5 and ABCG8 in three cell types: $\mathrm{CHO}-\mathrm{K} 1$ cells, nonpolarized cultured rat hepatocytes (CRL-1601 cells), and polarized hepatocytes (WIF-B cells). We provide evidence that ABCG5 and ABCG8 are physically associated in these cells and that they chaperone each other out of the ER en route to the plasma membrane. The transporters colocalized with each other in the ER and plasma membranes of stably expressing cultured hepatocytes and were present on the apical (canalicular) membranes of WIF-B cells. Collectively, these data indicate that an ABCG5/ABCG8 complex is assembled in the ER, moves through the Golgi complex, and is targeted to the apical surface of cultured cells, suggesting that ABCG5 and ABCG8 may function as a heterodimer on the apical surfaces of cells to export neutral sterols. To our knowledge this is the first direct demonstration that trafficking of an $\mathrm{ABC}$ halftransporter to the cell surface requires the presence of its dimerization partner.

All experiments in this study were performed using epitope-tagged versions of ABCG5 and ABCG8, since antibodies that recognize the native proteins are not available. Although we cannot exclude the possibility that epitope tagging of the proteins alters their behavior in cells, experiments using tagged and untagged versions of ABCG8 indicate that this possibility is unlikely. The exit of ABCG5 from the ER requires coexpression of ABCG8. Both tagged and nontagged forms of ABCG8 allow transport of ABCG5 to the plasma membrane. These results indicate that the formation of ABCG5/ABCG8 complex and its trafficking to the cell surface are not influenced by the presence of the epitope tag. Similarly, the substitution of a proline residue for arginine at residue 220 of ABCG8 had no effect on the half-life, processing, or formation of heterodimers in CHO-K1 cells.

Both ABCG5 and ABCG8 are N-glycosylated. When each transporter is expressed individually in cells, the added sugars do not undergo maturation and the proteins are more rapidly degraded. When the two proteins are coexpressed, higher-molecular weight, Endo $\mathrm{H}$-resistant, neuraminidase-sensitive forms of both proteins appear. The higher-molecular weight forms of the proteins have significantly longer half-lives, suggesting that formation of the complex between the two half-transporters in the ER promotes their transit from the ER to the trans-Golgi complex and thus diverts them from a degradative pathway. Cell fractionation and immunolocalization studies in cultured hepatocytes confirm that ABCG5 is retained in the ER unless ABCG8 is expressed in the same cells. These data indicate that the cellular localizations of the proteins are contingent on their oligomeric structure.
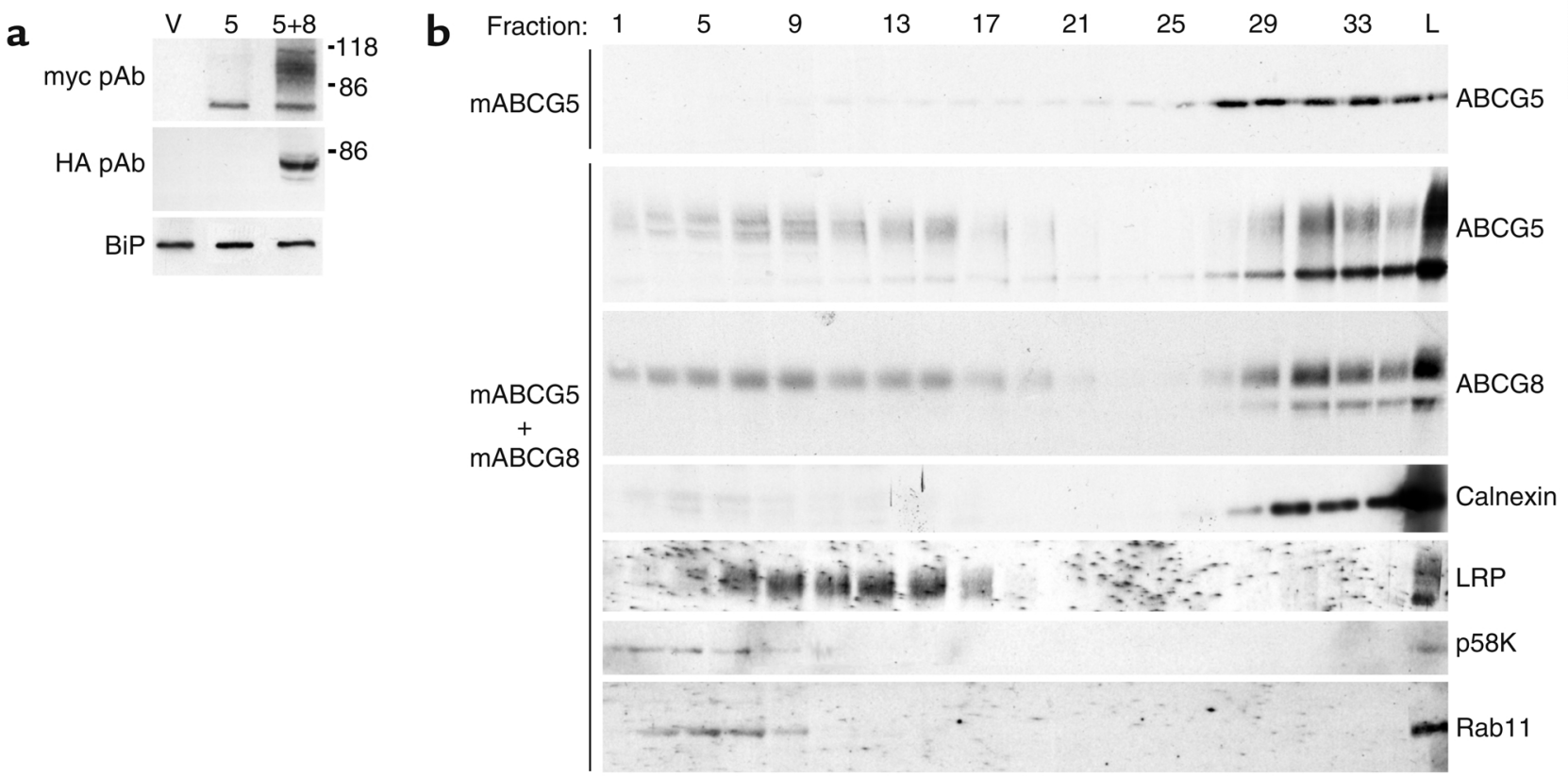

\section{Figure 4}

Relative levels and distribution of expression of ABCG5-myc and ABCG8-HA in CRL-1601 stably transfected with empty plasmids (V), ABCG5myc (5), or ABCG5-myc and ABCG8-HA (5+8). (a) Steady-state levels of immunoreactive ABCG5 and ABCG8 in CRL-1601 cells. Cell lysates $(5 \mu \mathrm{g})$ were subjected to SDS-PAGE and immunoblot analysis for expression of ABCG5 (top) or ABCG8 (middle). Immunoreactive ABCG5 and $A B C G 8$ were detected by enhanced chemiluminescence. (b) Fractionation of CRL-1601 cells that stably express ABCG5-myc alone or ABCG5-myc and ABCG8-HA. Cells were grown to 90\% confluence in 10-cm dishes. Postnuclear supernatants were prepared and fractionated as described in Methods. Fractions (20\%) were subjected to SDS-PAGE and immunoblot analysis for ABCG5-myc and ABCG8-HA. Separation of ER membranes from other organelles was confirmed by immunoblot analysis of marker proteins: calnexin (ER), LRP (plasma membrane and endosomes), p58K (Golgi complex), Rab11 (endosomes). 

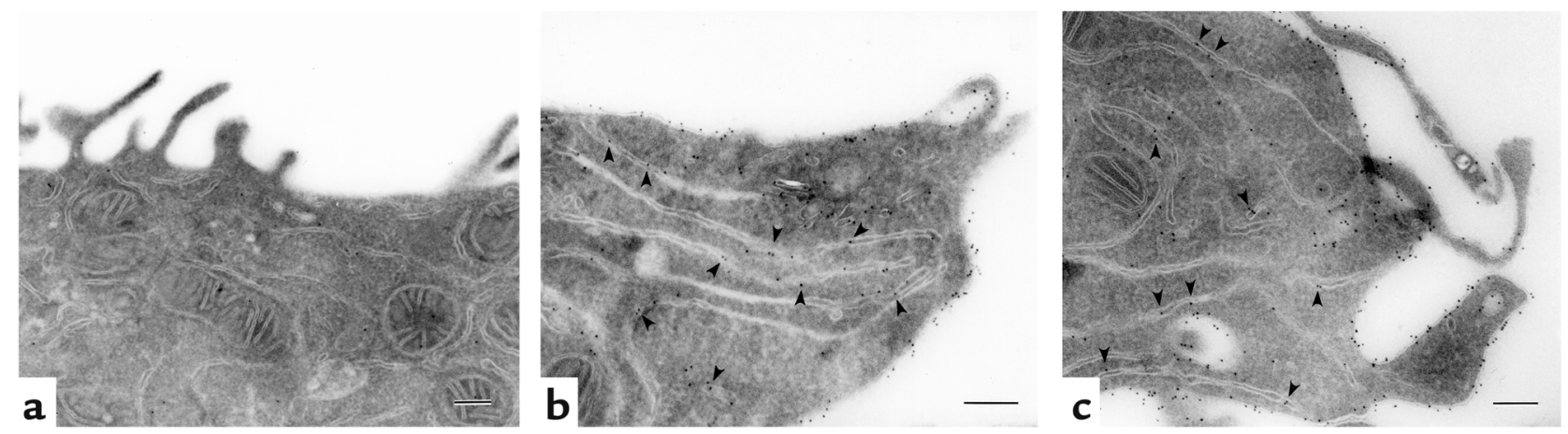

\section{Figure 5}

Immunolocalization of ABCG5 and ABCG8 in CRL-1601 cells by electron microscopy. Cells were cultured in 100-mm dishes and fixed and processed for immunoelectron microscopy as described in Methods. Localization of ABCG5 (a and b) and ABCG8 (c) in CRL-1601 cells containing empty plasmids (a) or ABCG5 and ABCG8 (b and $\mathbf{c})$. Scale bar $=2 \mu \mathrm{m}$.

The observation that mutations in either $A B C G 5$ or $A B C G 8$ cause an identical phenotype $(1,15)$ is also consistent with $A B C G 5$ and $A B C G 8$ forming a complex. Since ABC half-transporters contain only a single nucleotide-binding fold and six putative transmembrane segments, they must form homodimers or heterodimers to be active (14). Some half-transporters, such as TAP1 and TAP2, function as heterodimers; mutations in either gene result in the loss of peptide transport activity $(16,37)$. The four peroxisomal halftransporters (ABCD1-ABCD4) form homodimers and heterodimers in vitro (17), but the composition of the functional complexes of these proteins is not known. The ABCG subfamily members scarlet, brown, and white also function as heterodimers (38), whereas ABCG2 transports substrates when expressed alone in insect cells, suggesting that it functions as a homodimer (39). The finding that ABCG5 and ABCG8 coimmunoprecipitate indicates that the two proteins are associated in cells and suggests that they heterodimerize. The interaction between these two proteins is specific and is not a post-cell lysis artifact, since the half-transporters do not coimmunoprecipitate if lysates from cells expressing each half-transporter alone are mixed. Coimmunoprecipitation of the high-molecular weight, Endo $\mathrm{H}$-resistant forms of ABCG5-myc and ABCG8-HA is almost quantitative, further indicating that ABCG5/ABCG8 complex formation is required for ER exit.

A small fraction of expressed ABCG5 and ABCG8 also immunoprecipitates as homo-oligomeric complexes. For both proteins, only the lower-molecular weight forms are present in these complexes, indicating that if ABCG5 or ABCG8 form homodimers or other homo-oligomers, these complexes fail to exit the ER. Consistent with this interpretation, ABCG5-myc is not transported to the apical membrane in WIF-B cells if expressed alone. In contrast, ABCG2 appears to function as a homodimer and has been observed on the bile canalicular membrane (19). Further studies will be required to determine whether the ER-associated forms of ABCG5 and ABCG8 function as homodimers, represent nonspecific protein aggregates, or are mis-paired half-transporters that fail to achieve the appropriate conformation to escape the ER.

The observation that ABCG5-myc and ABCG8-HA reach the trans-Golgi complex only when the two proteins are coexpressed indicates that monomeric and multimeric homodimers of these proteins are specifically retained in the ER. The mechanism for ER retention of ABCG5 and ABCG8 is not known. Other oligomeric membrane proteins contain ER retention motifs that are masked when the proteins complex. For example, heterodimerization is required for the $\mathrm{T}$ cell antigen receptor (TCR), the $\mathrm{H} 2 \mathrm{a}$ subunit of the asialoglycoprotein receptor (ASGPR), and the $\mathrm{GABA}_{\mathrm{B}}$ receptor to exit the ER (40-42). Each subunit of these proteins contains a different ER retention motif (GLRILLLKV, EGHRG, and RXR for TCR, ASGPR, and $G_{A B A}$, respectively) that is masked by heterodimerization, allowing the protein complex to exit the ER. Only the RXR motif is present in mouse ABCG5 and ABCG8. One copy of this motif is conserved between the mouse and human proteins (residues 242-244 in ABCG5 and residues 196-198 in ABCG8), but the role of these residues in ER retention remains to be examined. Other mechanisms for the retention of improperly complexed oligomers include binding of proteins to ER chaperones that contain KDEL motifs, and thiol retention whereby the masking of cysteine residues by disulfide bonding allows proteins to exit the ER $(43,44)$. ABCG5 and ABCG8 contain numerous cysteine residues, but whether intramolecular or intermolecular disulfide bonds exist in the ABCG5/ABCG8 complex has not been examined. Additional studies will be required to determine the mechanisms by which ABCG5 and ABCG8 monomers, and perhaps oligomers, are recognized by the ER quality control system.

A potentially interesting observation is the finding of higher-molecular weight forms of ABCG5 and ABCG8 in the ER, as well as in the non-ER, fractions 
of cultured hepatocytes expressing both half-transporters. The presence of the mature forms in the ER fractions could represent contamination of these fractions with non-ER components of the cell, although none of the non-ER marker proteins are present in the ER fractions (Figure $4 \mathrm{~b})$. The fully mature, glycosylated forms of the proteins may have recycled from the Golgi complex compartment back to the ER, as has been described for SREBP cleavage-activating protein (SCAP) (45). More experiments will be required to determine the source of the mature forms of the halftransporters in the ER.

ABCG5 and ABCG8 colocalize at the cell surface in cultured hepatocytes, and immunoelectron microscopy confirms that the proteins are associated with the plasma membrane in these cells. The proteins are located predominantly on the apical plasma membrane, suggesting that ABCG5 and ABCG8 may participate directly in the transport of neutral sterols across the apical membrane into bile. It is not known whether neutral sterols are the primary transport substrate of ABCG5 and ABCG8 or whether another molecule is actively transported and sterol efflux is secondary. Phospholipids are the primary transport substrate for a number of other $A B C$ lipid transporters, and phospholipid transport and cholesterol transport into bile are tightly interrelated. For example, biliary cholesterol excretion is markedly reduced in mice lacking the phosphatidylcholine transporter ABCB4 (MDR2) (46). Sitosterolemic patients have a marked reduction in fractional excretion of biliary sterols but have normal biliary excretion of phospholipids (7). Conversely, overexpression of ABCG5 and ABCG8 in mice causes a selective increase in cholesterol excretion

\section{Figure 6}

Immunolocalization of ABCG5-myc in WIF-B cells. Cells were grown on glass coverslips, infected with virus expressing $A B C G 5$-myc alone or ABCG5-myc and $A B C G 8$, and processed for immunofluorescence microscopy. (a-c) Immunoreactive ABCG5-myc in cells infected with virus expressing $\beta$-gal (a), ABCG5-myc alone (b), or ABCG5-myc and ABCG8 (c). Coverslips containing cells expressing $A B C G 5$-myc alone $(\mathbf{d}-\mathbf{I})$ were incubated with anti-myc pAb and anti-BiP mAb (d-f), anti-myc mAb and anti-HA321 pAb (g-i), or anti-myc $m A b$ and anti-APN pAb (j-I). Colocalization is shown as a merged image $(\mathbf{f}, \mathbf{i}$, and $\mathbf{I})$. Immunoreactive ABCG5$\operatorname{myc}(\mathbf{m}, \mathbf{p}, \mathbf{s}$, and $\mathbf{v}), \operatorname{BiP}(\mathbf{n}), \operatorname{HA} 321(\mathbf{q}), \operatorname{APN}(\mathbf{t})$, mannosidase II (MII; $\mathbf{w})$, and their merges $(\mathbf{o}, \mathbf{r}, \mathbf{u}$, and $\mathbf{x})$ are shown in cells expressing ABCG5-myc and ABCG8.
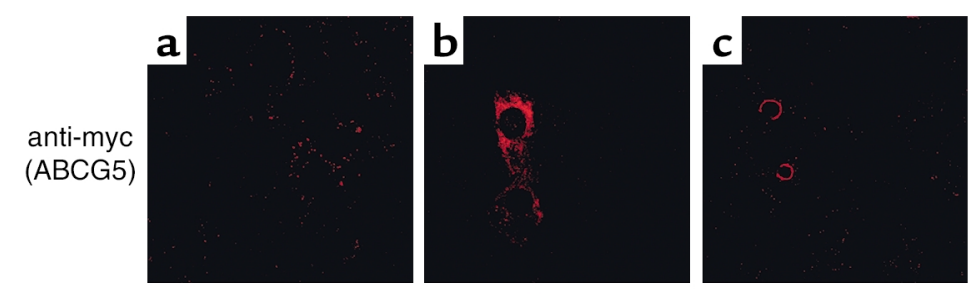

\section{WIF-B/ABCG5-myc}
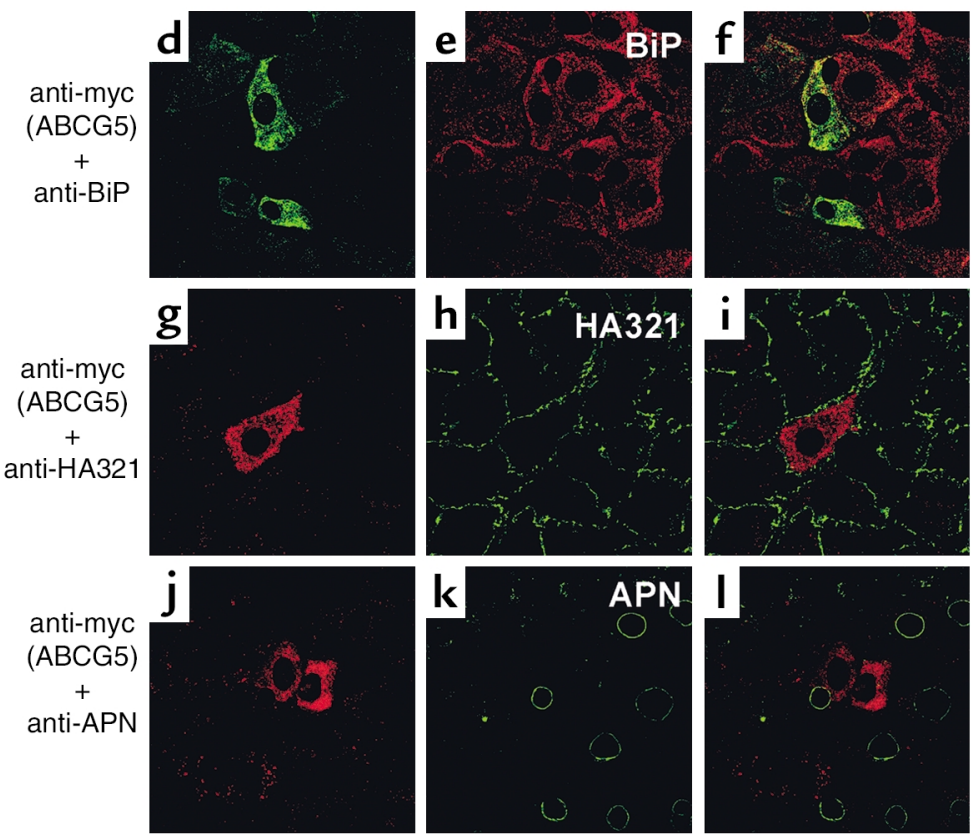

\section{ABCG5-Myc + ABCG8}
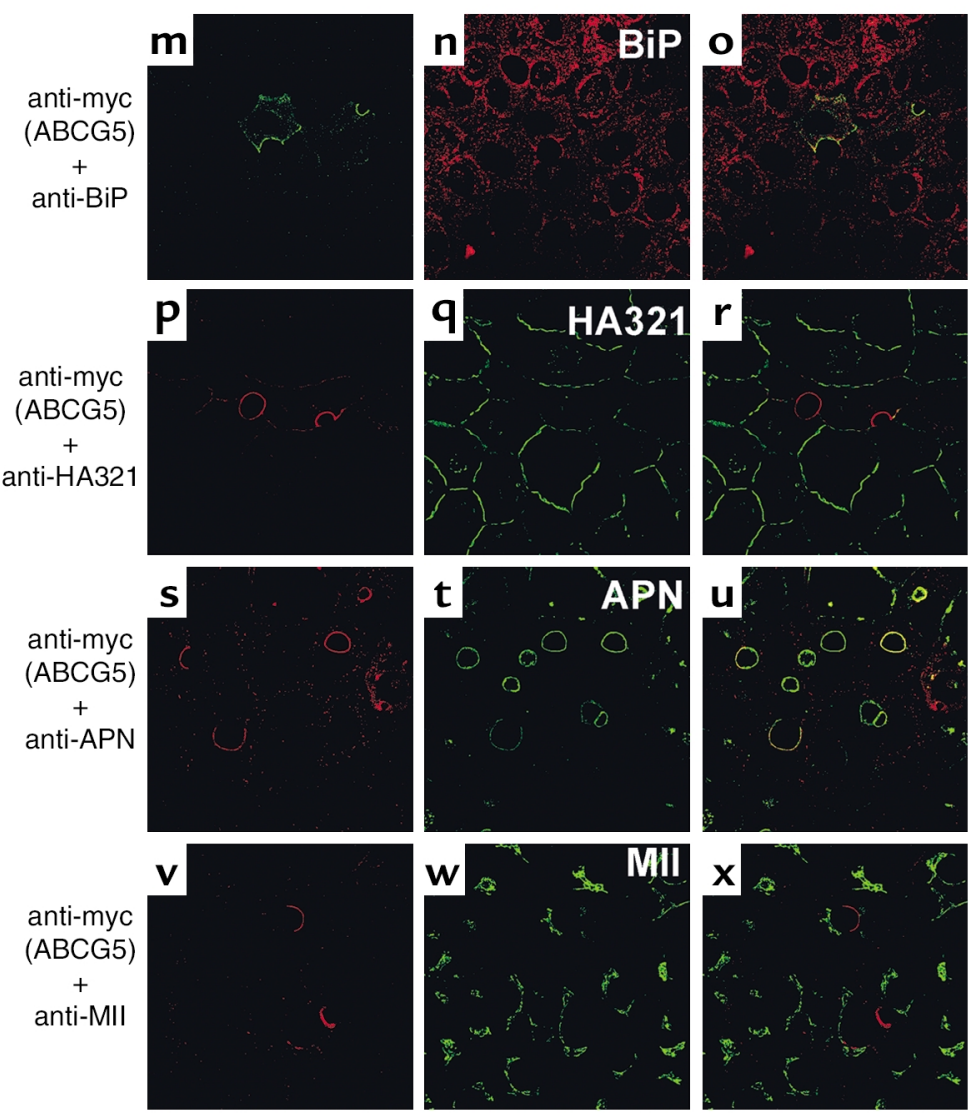
into the bile (47). These findings suggest that neutral sterols or a neutral sterol carrier protein, rather than phospholipids, are the primary transport substrate of ABCG5 and ABCG8.

The observation that ABCG5 and ABCG8 form a heterodimer does not exclude the possibility that either protein may form functional complexes with other ABC half-transporters. In Drosophila, white forms functional complexes with both scarlet and brown to transport different substrates (38). The mammalian ABCG transporter subfamily includes six known members. Although the tissue expression patterns of ABCG5 and ABCG8 differ from those of the other ABCG subfamily members, both ABCG1 and ABCG2 are expressed in the liver and intestine $(19,48,49)$. ABCG3 and ABCG4 are expressed at only low levels in the liver and intestine of mice (data not shown); the tissue distribution of expression of these proteins in humans is not known. Thus, it remains possible that ABCG5 and ABCG8 are not monogamous and that the half-transporters associate with other subfamily members to transport different substrates. The formation of dimer pairs between various members of this subfamily of proteins may permit transport of a more diverse array of substrates, or provide tissue- and/or substrate-specific control of solute transport.

\section{Acknowledgments}

We thank Ann Hubbard and Lita Braiterman for the generous gift of the WIF-B cells and helpful discussions. We thank Liangcai Nie, Jin Wang, and Lisa Beatty for excellent technical assistance; Kathleen Neill for assistance with the manuscript preparation; and David W. Russell and Richard Anderson for helpful discussions. This work was supported by the National Heart, Lung, and Blood Institute (POI HL2098), the W.M. Keck Foundation, the Perot Family Fund, and the Donald W. Reynolds Cardiovascular Clinical Research Center (Dallas, Texas, USA).

1. Berge, K.E., et al. 2000. Accumulation of dietary cholesterol in sitosterolemia caused by mutations in adjacent $\mathrm{ABC}$ transporters. Science. 290:1771-1775.

2. Lee, M.H., et al. 2001. Identification of a gene, ABCG5, important in the regulation of dietary cholesterol absorption. Nat. Genet. 27:79-83.

3. Bhattacharyya, A.K., and Connor, W.E. 1974. Beta-sitosterolemia and xanthomatosis. A newly described lipid storage disease in two sisters. J. Clin. Invest. 53:1033-1043.

4. Bjorkhem, I., Boberg, K., and Leitersdorf, E. 2001. Inborn errors in bile acid biosynthesis and storage of sterols other than cholesterol. In The metabolic and molecular bases of inherited disease. Volume II. C. Scriver, A. Beaudet, W. Sly, and D. Valle, editors. McGraw-Hill. New York, New York, USA. 2961-2988.

5. Salen, G., Ahrens, E.H., Jr., and Grundy, S.M. 1970. Metabolism of betasitosterol in man. J. Clin. Invest. 49:952-967.

6. Gould, R.G., Jones, R.J., LeRoy, G.V., Wissler, R.W., and Taylor, C.B. 1969. Absorbability of beta-sitosterol in humans. Metabolism. 18:652-662.

7. Miettinen, T.A. 1980. Phytosterolaemia, xanthomatosis and premature atherosclerotic arterial disease: a case with high plant sterol absorption, impaired sterol elimination and low cholesterol synthesis. Eur. J. Clin. Invest. 10:27-35.

8. Salen, G., et al. 1989. Increased sitosterol absorption, decreased removal, and expanded body pools compensate for reduced cholesterol synthesis in sitosterolemia with xanthomatosis. J. Lipid Res. 30:1319-1330.
9. Lutjohann, D., Bjorkhem, I., Beil, U.F., and von Bergmann, K. 1995. Sterol absorption and sterol balance in phytosterolemia evaluated by deuterium-labeled sterols: effect of sitostanol treatment. J. Lipid Res. 36:1763-1773.

10. Nguyen, L.B., et al. 1990. Decreased cholesterol biosynthesis in sitosterolemia with xanthomatosis: diminished mononuclear leukocyte 3-hydroxy-3-methylglutaryl coenzyme A reductase activity and enzyme protein associated with increased low-density lipoprotein receptor function. Metabolism. 39:436-443.

11. Salen, G., et al. 1992. Sitosterolemia. J. Lipid Res. 33:945-955.

12. Higgins, C. 1992. ABC transporters: from microorganisms to man. Annu. Rev. Cell Biol. 8:67-113.

13. Dean, M., and Allikmets, R. 1995. Evolution of ATP-binding cassette transporter genes. Curr. Opin. Genet. Dev. 5:779-785.

14. Dean, M., Hamon, Y., and Chimini, G. 2001. The human ATP-binding cassette (ABC) transporter superfamily. J. Lipid Res. 42:1007-1017.

15. Lu, K., et al. 2001. Two genes that map to the STSL locus cause sitosterolemia: genomic structure and spectrum of mutations involving sterolin- 1 and sterolin-2, encoded by $A B C G 5$ and $A B C G 8$, respectively. Am. J. Hum. Genet. 69:278-290.

16. Kleijmeer, M.J., et al. 1992. Location of MHC-encoded transporters in the endoplasmic reticulum and cis-Golgi. Nature. 357:342-344.

17. Liu, L.X., et al. 1999. Homo- and heterodimerization of peroxisomal ATP-binding cassette half-transporters. J. Biol. Chem. 274:32738-32743.

18. Mackenzie, S.M., Howells, A.J., Cox, G.B., and Ewart, G.D. 2000. Subcellular localisation of the white/scarlet $\mathrm{ABC}$ transporter to pigment granule membranes within the compound eye of Drosophila melanogaster. Genetica. 108:239-252.

19. Maliepaard, M., et al. 2001. Subcellular localization and distribution of the breast cancer resistance protein transporter in normal human tissues. Cancer Res. 61:3458-3464.

20. Repa, J.J., et al. 2002. Regulation of ATP-binding cassette sterol transporters, ABCG5 and ABCG8, by the oxysterol receptors, LXRalpha and beta. J. Biol. Chem. 277:18793-18800.

21. Cassio, D., Hamon-Benais, C., Guerin, M., and Lecoq, O. 1991. Hybrid cell lines constitute a potential reservoir of polarized cells: isolation and study of highly differentiated hepatoma-derived hybrid cells able to form functional bile canaliculi in vitro. J. Cell Biol. 115:1397-1408.

22. Ihrke, G., et al. 1993. WIF-B cells: an in vitro model for studies of hepatocyte polarity. J. Cell Biol. 123:1761-1775.

23. Sakai, J., et al. 1998. Molecular identification of the sterol-regulated luminal protease that cleaves SREBPs and controls lipid composition of animal cells. Mol. Cell. 2:505-514.

24. Tokiwa, G., Tyers, M., Volpe, T., and Futcher, B. 1994. Inhibition of G1 cyclin activity by the Ras/cAMP pathway in yeast. Nature. 371:342-345.

25. Lu, K., et al. 2002. Molecular cloning, genomic organization, genetic variations, and characterization of murine sterolin genes Abcg5 and Abcg8. J. Lipid Res. 43:565-578.

26. Sakai, J., et al. 1997. Identification of complexes between the COOHterminal domains of sterol regulatory element-binding proteins (SREBPs) and SREBP cleavage-activating protein. J. Biol. Chem. 272:20213-20221.

27. Graf, G.A., Connell, P.M., van der Westhuyzen, D.R., and Smart, E.J. 1999. The class B, type I scavenger receptor promotes the selective uptake of high density lipoprotein cholesterol ethers into caveolae. J. Biol. Chem. 274:12043-12048.

28. Hammond, C., and Helenius, A. 1994. Quality control in the secretory pathway: retention of a misfolded viral membrane glycoprotein involves cycling between the ER, intermediate compartment, and Golgi apparatus. J. Cell Biol. 126:41-52.

29. Willnow, T.E., Armstrong, S.A., Hammer, R.E., and Herz, J. 1995. Functional expression of low density lipoprotein receptor-related protein is controlled by receptor-associated protein in vivo. Proc. Natl. Acad. Sci. USA. 92:4537-4541.

30. Sai, Y., Nies, A.T., and Arias, I.M. 1999. Bile acid secretion and direct targeting of mdr1-green fluorescent protein from Golgi to the canalicular membrane in polarized WIF-B cells. J. Cell Sci. 112:4535-4545.

31. Tokuyasu, K.T. 1980. Immunochemistry on ultrathin frozen sections. Histochem. J. 12:381-403.

32. Aoki, K., Barker, C., Danthinne, X., Imperiale, M.J., and Nabel, G.J. 1999. Efficient generation of recombinant adenoviral vectors by Cre-lox recombination in vitro. Mol. Med. 5:224-231.

33. Cummings, R.D., et al. 1983. Biosynthesis of N- and O-linked oligosaccharides of the low density lipoprotein receptor. J. Biol. Chem. 258:15261-15273.

34. Kornfeld, R., and Kornfeld, S. 1985. Assembly of asparagine-linked oligosaccharides. Annu. Rev. Biochem. 54:631-664.

35. Song, W., Apodaca, G., and Mostov, K. 1994. Transcytosis of the polymeric immunoglobulin receptor is regulated in multiple intracellular compartments. J. Biol. Chem. 269:29474-29480.

36. Scott, L.J., and Hubbard, A.L. 1992. Dynamics of four rat liver plasma membrane proteins and polymeric IgA receptor: rates of synthesis and 
selective loss into the bile. J. Biol. Chem. 267:6099-6106.

37. Kelly, A., et al. 1992. Assembly and function of the two ABC transporter proteins encoded in the human major histocompatibility complex. Nature. 355:641-644.

38. Sullivan, D.T., and Sullivan, M.C. 1975. Transport defects as the physiological basis for eye color mutants of Drosophila melanogaster. Biochem. Genet. 13:603-613.

39. Ozvegy, C., et al. 2001. Functional characterization of the human multidrug transporter, ABCG2, expressed in insect cells. Biochem. Biophys. Res. Commun. 285:111-117.

40. Shenkman, M., Ehrlich, M., and Lederkremer, G.Z. 2000. Masking of an endoplasmic reticulum retention signal by its presence in the two subunits of the asialoglycoprotein receptor. J. Biol. Chem. 275:2845-2851.

41. Margeta-Mitrovic, M., Jan, Y.N., and Jan, L.Y. 2000. A trafficking check-

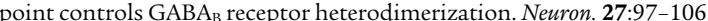

42. Bonifacino, J.S., Cosson, P., and Klausner, R.D. 1990. Colocalized transmembrane determinants for ER degradation and subunit assembly explain the intracellular fate of TCR chains. Cell. 63:503-513.

43. Munro, S., and Pelham, H.R. 1987. A C-terminal signal prevents secretion of luminal ER proteins. Cell. 48:899-907.
44. Reddy, P.S., and Corley, R.B. 1998. Assembly, sorting, and exit of oligomeric proteins from the endoplasmic reticulum. Bioessays. 20:546-554.

45. Nohturfft, A., DeBose-Boyd, R.A., Scheek, S., Goldstein, J.L., and Brown, M.S. 1999. Sterols regulate cycling of SREBP cleavage-activating protein (SCAP) between endoplasmic reticulum and Golgi. Proc. Natl. Acad. Sci. USA. 96:11235-11240.

46. Smit, J.J., et al. 1993. Homozygous disruption of the murine Mdr2 P-glycoprotein gene leads to a complete absence of phospholipid from bile and to liver disease. Cell. 75:451-462.

47. Yu, L, et al. 2002. Overexpression of ABCG5 and ABCG8 promotes biliary cholesterol secretion and reduces fractional absorption of dietary cholesterol. J. Clin. Invest. 110:671-680. doi:10.1172/JCI200216001.

48. Klucken, J., et al. 2001. ABCG1(ABC8), the human homolog of the Drosophial white gene, is a regulator of macrophage cholesterol and phospholipid transport. Proc. Natl. Acad. Sci. USA. 97:817-822.

49. Kennedy, M.A., et al. 2001. Characterization of the human ABCG1 gene: liver $\mathrm{X}$ receptor activates an internal promoter that produces a novel transcript encoding an alternative form of the protein. J. Biol. Chem. 276:39438-39447. 